\title{
Phase shift via polarizer in an heterodyne interferometer
}

\section{A. Patra, Alika Khare}

A. S. Patra, Alika Khare, "Phase shift via polarizer in an heterodyne interferometer," Proc. SPIE 9664, Ninth International Topical Meeting on Education and Training in Optics and Photonics, 96642E (24 October 2005); doi: $10.1117 / 12.2207543$ 
Ref ETOP008

Phase shift via polarizer in an heterodyne interferometer

\author{
A. S. Patra and Alika Khare ${ }^{1}$
}

${ }^{1}$ Department of Physics, Indian Institute of Technology Guwahati, Guwahati- 781039, India.

Email: alika@iitg.ernet.in

\begin{abstract}
Polarization interferometry is normally not a regular topic in the class room at graduate level. Therefore a polarization heterodyne interferometer similar to a Michelson interferometer using polarizing components is discussed in this paper to demonstrate the interference of two orthogonal polarized beams. The measurement of the phase shift as a result of orientation of polarizer is performed by recording corresponding fringe shift. The theoretical expression is also presented using Jones calculus for the fringe shift as a function of azimuth of the polarizer. The experimental set-up is simple and can be very well incorporated in the teaching laboratory.
\end{abstract}

\title{
Summary
}

Polarization interferometry is normally not talked about at graduate level neither in theory and nor in laboratory. The students go with the concept that two different polarized light always result in a fringe free pattern. In this paper, a simple configuration similar to Michelson interferometer using polarized components is reported for the interference of two orthogonally polarized light in the class room. The measurement of the phase shift as a result of orientation of polarizer is performed. The theoretical expression is also presented for the fringe shift as a function of azimuth of the polarizer.

The common method to realize relative phase shift in an optical beam of the Michelson interferometer in the class room is by physically changing the optical path of one of the arm of the interferometer either by translating mirrors or by introducing the optical surfaces in the form of glass plate ${ }^{1}$ in a controlled way. This phase shift is observed either in the form of fringe shift or some modification in the interference pattern. With one or more rotating polarizing components viz; polarizer, quarter wave plate or half wave plate, phase shift can also be observed ${ }^{2-5}$. In this paper, we report the effect of azimuth angle of polarizer in the output plane of two orthogonally polarized interfering beams from a heterodyne interferometric setup. The experimental set-up presented below is simple and can be handled by the graduate students independently.

\section{Experimental setup and Theory}

A collimated laser beam was launched into an interferometric setup, as shown in fig.1. The polarizer $\mathrm{P}_{1}$ was aligned at $45^{\circ}$ ensures $50-50$ splitting of light from polarizing beam splitter (PBS). The transmitted beam (only p-polarized light) from PBS passed through Quarter Wave Plate $Q_{2}\left(\mathrm{QWP}\right.$ at $45^{\circ}$ ) converted into right circularly polarized (r.c.p.) light. The r.c.p. beam reflected from mirror $\mathrm{M}_{2}$, became left circularly polarized (I.c.p.) and passed back through $\mathrm{Q}_{2}$. It turned into s-polarized light and reflected completely from PBS. The reflected beam (only s-polarized light) after passing through $Q_{1}, M_{1}$ and 
back from $Q_{1}$ is converted into p-polarized light finally transmitted through PBS. So two orthogonal linearly polarized beams came out at the output of PBS. These orthogonal polarized beams produced fringe free pattern. To obtain the fringes, beams were passed through the third $\mathrm{QWP} \mathrm{Q}_{3}$ and then through the polarizer $\mathrm{P}$ and the interference pattern was finally recorded on to CCD.

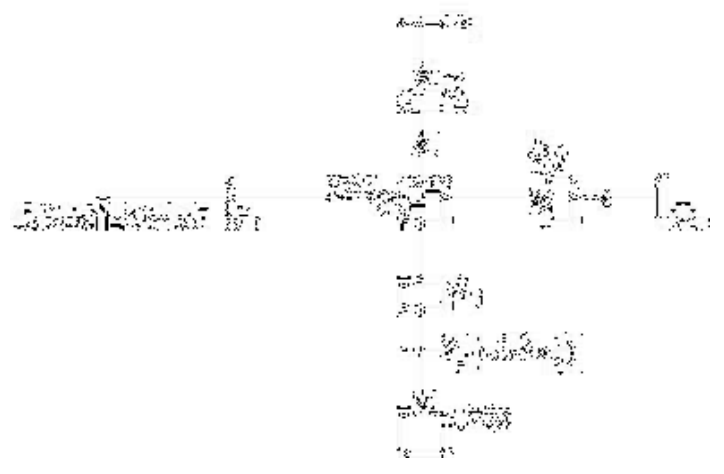

Fig.1. Experimental setup for phase shifting interferometry; $Q_{1}, Q_{2}$ and $Q_{3}$ : quarter wave plates, $M_{1}, M_{1}$ : mirrors, $P_{1}, P_{2}$ : polarizers, PBS: polarizing beam splitter, CCD: charge coupled device.

For analyzing the effect of the azimuth angle of polarizer (and hence the phase shift) onto the interferograms, $Q_{1}$ to $Q_{3}$ were aligned at $45^{\circ}$ with respect to polarization plane of the incident beam on the respective QWP's and $P_{2}$ was rotated. The resultant intensity at output plane can be shown to be given by ${ }^{6,7}$

$\mathrm{I}=\frac{a^{2}}{4}[1+\operatorname{Sin}(2 \theta+\mu y)]$

Where spatial frequency $\mu=\frac{2 \pi \operatorname{Sin} \theta_{1}}{\lambda}, \theta_{1}$ is the angular separation between the two interfering beams which can be controlled by the tilt of mirror $M_{2}, M_{1}$ being kept for the normal incidence. From above equation, if the analyzer is rotated by $\theta$ then $2 \theta$ phase difference is introduced between the two interfering beams and there will be corresponding fringe shift in the interference pattern, which is recorded onto CCD. The condition for minima for the $n$th order fringe from equation (1) is $2 \theta+\mu y=(2 n \pm 1 / 2) \pi$. If the spatial frequency $\mu$ is kept constant then the location of fringe for any particular order will change linearly with respect to the azimuth angle $\theta$. The fringe shift is used to measure the corresponding phase shift as a function of $\theta$ which will also vary linearly with $\theta$.

\section{Results}

The recorded interference patterns for $\theta=0^{\circ}, 45^{\circ}, 90^{\circ}, 135^{\circ}, 180^{\circ}$ on to CCD are shown in the fig.2. (a), (b), (c), (d), (e) respectively. From fig.2, it is clearly observed that $180^{\circ}$ rotation of the analyzer gives one fringe shift confirming an additional phase difference 
of $2 \pi$ is developed between the two interfering orthogonal beams. The plot of measured phase shift versus the azimuth of analyzer $(\theta)$ is shown in fig. 3 The plot confirm that the phase shift goes linearly with the azimuth $\theta$ of analyzer $P_{2}$.

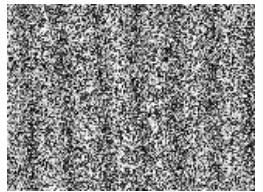

(a)

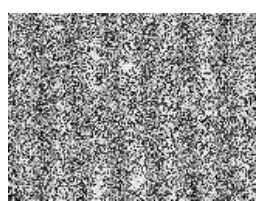

(d)

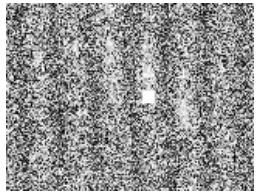

(b)

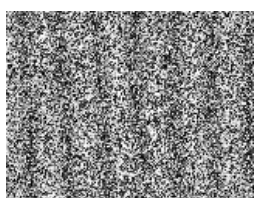

(e)

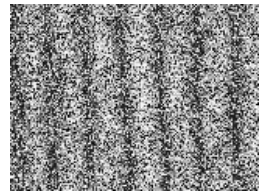

(c)

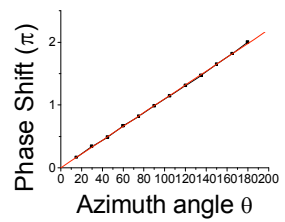

(f)

Fig.2. Interference pattern recorded onto CCD for (a) $\theta=0^{\circ}$, (b) $\theta=45^{\circ}$, (c) $\theta=90^{\circ}$, (d) $\theta=135^{\circ}$, (e) $\theta=180^{\circ}$. (b) variation of phase shift as a function of azimuth of output polarizer.

\section{Conclusion}

We have experimentally observed the phase shift between the two orthogonal interfering beams using the set-up similar to Michelson interferometer and a quarter wave plate and an analyzer (rotating) at the output of the interferometer. We have recorded the interference pattern on to CCD and measured the fringe shift. The experiment can very well be performed using a photodiode mounted on the translation stage in place of CCD.

\section{Acknowledgement}

This work is partially financed by CSIR, New Delhi, India, Scheme no. 3(831)/98-EMR-II.

\section{References}

1. D. Malacaara, Optical Shop Testing (John Wiley and Sons, New York, (1992)

2. P. Hariharan and M. Roy, Optics communications 126, 220-222 (1996).

3. Asundi A., Liu T., and Boay C. G., Applied Optics 38, 5931-5935 (1999).

4. Kothiyal M.P. and Delisle C., Optica Acta 33 787-793 (1986.).

5. Chyba T.H., Wang L.J., Mandel L., and Simon R., Optics Letter,13 562-564 (1998).

6. A S Patra and Alika Khare, 'Studies on two beam heterodyne interferometer', J of Optical tech (in press, to appear in vol. 72, no. 12, 2005).

7. D S Kilger, J W Lewis and C E Randel, Polarised light in optics, Acdemic press (1990) 